\title{
Unmanned autonomous vessels and the necessity of human-centred design
}

\author{
Cecilie Kristoffersen ${ }^{1}$ \\ ${ }^{1}$ Norwegian University of Science and Technology \\ cille.kristoffersen@gmail.com
}

\begin{abstract}
The introduction of an unmanned autonomous vessels is said to reduce the high percentage of human error in the shipping industry. Still, human errors will be present, from the design of automation's algorithms to the operator monitoring the vessels in the Shore Control Center. However, there is a concern that when the system gets more complex so does the human errors. Operators are moved from the sea to the shore and must change how they obtain their situation awareness, as all sensory information no longer is available. The loss of situation awareness is linked to be a cause of human errors in accidents. This paper argues that by making use of human-centered design methods and designing for the operator's situation awareness, in the development of unmanned autonomous vessels and their Shore Control Centers, one can reduce human error further. The paper concludes with five guidelines for enhancing the operator's situation awareness in the Shore Control Center. The guidelines are about keeping the operator in the loop, replace lost sensory information, available information, automation transparency and the indication of autonomy level.
\end{abstract}

Keywords: human centred design, cognitive capabilities, complex systems, human error, unmanned autonomous vessels

\section{Introduction}

When developing new technology, it is easy and unfortunately quite common, to neglect the user's needs and limits. The mentality that the "user can adapt to the new system," also known as technology-driven design, can be catastrophic for the users of complex systems within domains like aviation, shipping, medicine and nuclear powerplants (Endsley \& Jones, 2012; Oviatt, 2006; Rothblum, 2000). The "user adapts" mentality has made systems so complex that it is mentally challenging and draining for users to utilize them (Endsley \& Jones, 2012), often resulting in accidents attributed to human error. Between $75-96 \%$ of accidents are said to be due to human error within the shipping industry. Even though the accident rate has been reduced considerably after the introduction of automation, technology advances and better ship design, the rate of human error still stand strong (Allianz Global Corporate \& Specialty SE (AGCS), 2018; Porathe, Hoem, Johnsen, Rødseth, \& Fjørtoft, 2018). 
For the last three decades or so, literature has suggested using Human-Centred Design (HCD) methods to improve the human-machine interaction regarding automation in different industries (Endsley \& Jones, 2012; Inagaki, 2006; Oviatt, 2006; Rothblum, 2000). To design technological systems that consider human limits and strengths, one must create a "system that is designed to support situation awareness (SA)" (Endsley \& Jones, 2012, p. 5). In aviation, the pilot's SA has gained attention in the aftermath of accidents. Subsequently increased focus on SA has made flying safer (Wiegmann and Shappell, 2007). A study conducted by Endsley (1995) on aviation accidents attributed to be caused by human error, found that $88 \%$ of these accidents was due to problems with SA. The accident rate attributed to human error within aviation is high. According to Wiegmann and Shappell (2007), if one had accepted the accident rate attributed to human error as it is today, the overall frequency of accidents would increase. The reason being that the increasing number of commercial flights and the number of accidents correlates. The changes within the shipping industry have been slower. The focus still seems to be on technological-driven design since it is more affordable than using HCD-methods, which implicates involving users and multiple iterations after user testing. Shipping makes up $90 \%$ of all global trade, and ships are built larger for each decade that passes (AGCS, 2018), so it is equally important to reduce the human error rate within the shipping industry.

The shipping industry is moving into a new era with unmanned and autonomous ships, which not only will reduce emissions (Kongsberg Gruppen, 2017; Tinlund Ryste, 2018), but will also reduce the accidents attributed to human error substantially (AGCS, 2018; Porathe et al., 2018). Porathe et al. (2018) argue that the individual is still in the loop, ranging from the ones that design the system to the operators monitoring the ship. Thus, the probability for new unknown human errors is high. The shipmaster or operator will no longer be onboard the ship, but in Shore Control Centers (SCC), observing up to eight ships at the time (Kongsberg Gruppen, 2017; Porathe, Prison, \& Man, 2014). He must be prepared to step in if emergencies or unexpected events happen that the automation can't handle. It can be challenging for the operator to gain good SA when observing the ships in the SCC. With monitors providing only visual and audio cues (Porathe et al., 2014). If the captain is onboard the ship he will use all his senses to understand the sea, and how the ship reacts to the sea and weather. Without a wellmade solution to replace the sensory information the operator risks a loss of SA (Endsley \& Jones, 2012; Porathe et al., 2014).

Based on a comprehensive literature review, this paper will explore how the incorporation of HCD methods in the development of autonomous vessels and SCC can lower human errors and give the operator a good SA.

\section{Method}

The research field on unmanned autonomous vessels is quite young. Full-scale projects, such as Yara Birkeland in Norway, have just started. The same goes for the research on Shore Control Centers and the effect of moving the operator from sea to shore will have on the operator. The EU project MUNIN (Porathe et al., 2014), a three-year research project, investigated the technology and resources needed for a Trans-Atlantic voyage using a SCC. The literature on unmanned and autonomous vessels and how it affects the user is limited. Therefore, this literature review is informed by other fields such as situation awareness, human errors within shipping and other domains, automation and human-centered design. There is a lot of research and theories on areas such as situation awareness and automation from other domains than shipping, especially within aviation. Aviation has a long history of unmanned aerial vessels (UAV) and accidents (Whitlock, 2014). Looking at studies of these UAV accidents where the 
operator experience loss of situation awareness and human error was a factor can help understand possible factors that may affect an operator in a SCC.

Literature, both books, articles and webpages were found in online libraries, such as NTNU's Oria, Mendeley and Google Scholar, and reviewed against several criteria. Some of the criteria were that the research was published the last 30 years and had serval citations. Literature suggested by Thomas Porathe, one of the MUNIN researchers, was also reviewed. Secondary sources were found in the reviewed papers' reference list and used to expand knowledge on topics.

This literature review starts with an introduction to unmanned and autonomous vessels, as well as the SCC. From there it looks at situation awareness, human limits and which preconditions that may cause human cause errors. It will conclude in a discussion on how human-centered design can reduce human errors in the development of SCC for unmanned autonomous vessels.

\section{Autonomous vessels}

Shipping has evolved over the last centuries from mechanical power to mass production and computerized control in the 1970s. We are now entering a new era of shipping, "Shipping 4.0", where unmanned and autonomous ships can be game changers (Ø. Rødseth, 2018). The financial, sustainable and safety benefits of unmanned autonomous ships are substantial. YARA is one of the companies that are exploring these benefits together with Kongsberg Maritime on the YARA Birkeland project. YARA Birkeland is going to be the world's first zero emission autonomous container ship when completed. By being fully electric and traveling at a slower speed, the ship is planned to replace 40.000 journeys done by diesel trucks annually from 2021 . That will reduce $\mathrm{CO} 2$ emissions by 750 tons. The ship will travel between Herøya, Brevik and Larvik, initially with a captain and crew onboard to assure that the transition to a fully autonomous and unmanned ship goes smoothly. Both the reduction of personnel and trucks will contribute to reduce the transportation cost for YARA considerably (Kongsberg Gruppen, 2017; Stensvold, 2018). Kongsberg Maritime (2017) will develop SCC where operators can handle emergencies, exceptions, condition monitoring and surveillance on surroundings for YARA Birkeland. The MUNIN project (Porathe \& Man, 2013, 2014; Porathe et al., 2014) developed a concept for a SCC for Trans-Atlantic voyages, where operators can remotely monitor several vessels on multiple monitors - and take over control in parts of the voyage that the automation cannot handle by itself.

This paper focus on unmanned autonomous vessels. "Unmanned" and "autonomous" are partly independent definitions. An unmanned vessel can be remotely controlled or controlled by autonomy. A vessel is autonomous when it is managed by a computerized control system, making decisions by itself. It can be manned by a crew or unmanned (Ø. J. Rødseth \& Nordahl, 2018; Tinlund Ryste, 2018). YARA Birkeland is going to be both unmanned and autonomous, with a SCC overlooking it, and operate on a constrained autonomous level.

\subsection{Level of automation}

Autonomy has different levels, ranging from how much the human versus the computer is in control. There are several models in the literature that describes the different levels of automation. Some scholars have attempted to make a general model for all kind of automation, while other models fit a specific domain. Inagaki (2006) points out that each model should be domain specific, drawing examples from drivers of self-driving cars and pilots vs. autopilots in 
aviation. There is a difference in the user groups experience and training, as well as in the time criticality to make a decision. For example, when a pilot receives a collision warning against terrain or another aircraft, the pilot usually has enough time to grasp the situation and react. Drivers of self-driving cars with forwarding vehicle collision warning have at most a few seconds, and with less training than the pilots, the driver struggles to react in time (Inagaki, 2006). As for the autonomous vessels, the time criticality differs in nature from aviation and driving, because the ship itself has a slower reaction time. On the other hand, the ship operator has a higher possibility to anticipate collisions than pilots and drivers. Inagaki (2006) argues against transferring the models of autonomy from self-driving cars to other domains because domains have different requirements. Unmanned autonomous vessels have copied models of autonomy levels from other domains. Norwegian Forum for Autonomous Ships has made a model that combines serval models and made it domain dependent to autonomous vessels.

Table 1 show the possible levels of autonomy, where the shaded cells indicate that a SCC is needed. "Constrained autonomy" is the level of autonomy that seems most likely to be used in the foreseeable future, as it solves the "standard" problems by itself under supervision from an operator in the SCC (Porathe et al., 2018).

Table 1. Levels of autonomy (Ø. J. Rødseth \& Nordahl, 2018). The shaded cells indicate that the vessel will be monitored and/or controlled from shore.

\begin{tabular}{|c|c|c|c|}
\hline & $\begin{array}{c}\text { Continuously } \\
\text { manned bridge }\end{array}$ & $\begin{array}{c}\text { Unmanned bridge, } \\
\text { crew on board }\end{array}$ & $\begin{array}{c}\text { Unmanned bridge, no } \\
\text { crew on board }\end{array}$ \\
\hline $\begin{array}{c}\text { Operator } \\
\text { controlled }\end{array}$ & Direct control & Remote control & Remote control \\
\hline Automatic & Automatic control & Automatic control & Automatic control \\
\hline $\begin{array}{c}\text { Partly } \\
\text { autonomous }\end{array}$ & Partly autonomous & Partly autonomous & Partly autonomous \\
\hline $\begin{array}{l}\text { Constrained } \\
\text { autonomous }\end{array}$ & - & $\begin{array}{c}\text { Constrained } \\
\text { autonomy }\end{array}$ & Constrained autonomy \\
\hline $\begin{array}{c}\text { Full } \\
\text { autonomous }\end{array}$ & - & - & Full autonomous \\
\hline
\end{tabular}

\section{Situation awareness}

In 1988 Mica Endsley coined the term situation awareness (SA) to describe the pilots' internal model of the world around him, and how that information affected him now and in the future. SA applies not only to pilots but to all operators of complex systems - such as in nuclear plants control rooms, military UAV control rooms or SCC. SA is often referred to as "ship sense" in the shipping industry (Porathe et al., 2014). The formal definition of SA is:

"The perception of the elements in the environment within a volume of time and space, the comprehension of their meaning, and the projection of their status in the near future" (Endsley, 1988, p. 1).

The definition of SA can be broken down into three levels (Endsley \& Jones, 2012):

$\circ$ Level $1 \mathrm{SA}$ - the perception of the elements in the environment.

- Level $2 \mathrm{SA}$ - comprehension of the current situation.

- Level $3 \mathrm{SA}$ - projection of future status. 
Each level builds on the former one. Obtaining a good sense of SA is done by fulfilling each level that aid to reach a specific goal. The goal can for instance be to get from A to B or navigate a busy fjord while avoiding small boats and canoes. One incomplete level will affect the next level and result in poor SA. Endsley and Jones (2012) write that in complex systems like aviation, nuclear plants and shipping, acquiring a good SA is arduous and requires an extensive amount of training. These complex systems often rely heavily on electronic displays and their read-outs. Experienced and trained operators obtain an immense amount of relevant information from perceiving and sensing their environment. An experienced shipmaster can feel the movement and vibration from the ship as it moves through the waves - and adjust the ship to avoid damage accordingly.

The working memory is our biggest biological challenge when it comes to obtaining SA. With a capacity of 5 to 9 "chunks" of information it creates a bottleneck for the perceived information and will decay if it is not actively worked on (Endsley \& Jones, 2012). Another central bottleneck for SA is attention and perception. "The limit on how many elements one person can pay attention to at one time affects how much information a person can process" (Endsley \& Jones, 2012, p. 20). When designing to lessen the burden on the mental bottleneck, one should utilize audio and visual information together as these are easier processed simultaneously than e.g. speaking or writing since speaking and writing use the same processing path in the brain (Endsley \& Jones, 2012). To compensate for limitations in working memory and attention, humans use mental models from their long-term memory. Mental models are "a systematic understanding of how something works" (Endsley \& Jones, 2012, p. 21). For example, when a person wants to cook using a stove, the mental model provides an understanding of how the stove works, which buttons to press in the correct order and an expectation on how the stove reacts after his interaction.

Humans have limits, both mental and physical. Our mental capacity is easily affected by what Endsley and Jones (2012) define as the "SA demons". The major SA demons are: Attention tunnelling, requisite memory trap, workload, anxiety, fatigue, and other stressors, data overload, misplaced salience, errant mental models and the out-of-the-loop syndrome

SA demons appear in complex domains and in many different systems and environments, and it cripples the operators SA.

These demons hinder the development of gaining good SA, thus increasing the risk for human errors. It is important to design to avoid these demons and to limit their hold on the operators SA in order to avoid human errors.

\subsection{Out-of-the-loop syndrome}

When it comes to automation, it is essential to notice one specific SA demon, namely the outof-the-loop syndrome. Endsley and Kiris (1995) linked two significant issues between the outof-the-loop performance and implementation of automation: "the loss of manual skill and loss of awareness of the state and process of the system"(Endsley \& Kiris, 1995, p. 381). The outof-the-loop syndrome occurs when the operator is not in tune with what the automated system is doing, or his attention is on other parts of the system causing him to miss significant information. This lowers the SA, and the operator ends up using an excessive amount of time to try regaining a mental picture of the situation (Endsley \& Jones, 2012; Endsley \& Kiris, 1995). The automation can, by poor design, hide information that seems irrelevant but is vital in order for the operator to understand the problem if the system fails. An automation system 
done right will help the operator handle more than one thing at once, lower the mental workload and still be kept in the loop (Endsley \& Jones, 2012; Endsley \& Kiris, 1995).

Feedback is essential to keep the operator in the loop, to know if his request has been received or that required action is done correctly (Endsley \& Kiris, 1995). Otherwise, a misunderstanding of the automation can happen, and the wrong action is performed (Endsley \& Jones, 2012). Feedback can come from the system interface, but also from other sensory information, e.g. vibration and muscle memory. Feedback solely from visual cues can inhabit the operator from getting a full picture of the situation (Endsley \& Kiris, 1995).

\subsection{SA and Unmanned Vessels}

Remote-control stations, such as the SCC, have challenges when it comes to supporting the operators' SA. The operators need for SA is the same whether he is on board the ship or not. Endsley \& Jones (2012) explain that gaining and maintaining SA is challenging, especially since the operator no longer is on site. Now he must gain SA trough monitors and data from the unmanned vessel. In remote control stations for UAV and Unmanned Ground Vehicle the importance of considering human factors in the design and development process has frequently been neglected (Endsley \& Jones, 2012). This has led to an even higher accident rate attributed to human error for military UAVs (Endsley \& Jones, 2012; Manning, Rash, LeDuc, Noback, \& McKeon, 2004). Manning et al. (2004) analyzed accident reports of military UAVs that occurred in the period 1995 to 2003 and discovered that the UAVs had a higher percentage of human errors than manned flying. These human errors occurred because of poor SA, fatigue, high or low workload and how the remote-control center was not designed to support the operator needs.

The first challenge when designing and developing an SCC is the loss of the operator's direct sensory information. To compensate for this loss with visual cues, e.g., multiple monitors, the risk of visual overload and cognitive bottlenecks increases. That makes gaining and maintaining SA difficult and the operator risk to suffer from the out-of-the-loop syndrome (Endsley \& Jones, 2012). The second challenge is that the mental models used on handling the ship are no longer available. The operators in the SCC then need to build new mental models to support a high level of SA (Porathe et al., 2014). With no mental models for monitoring and controlling the unmanned autonomous vessel, the operator will experience a high workload while trying to gain the full picture.

\section{Human error}

The individual is at their best when they can solve problems and be creative. As Endsely state:

"The role of the human operator has changed dramatically. Instead of performing tasks, the human's job has become that of a monitor over an automated system - a role people are not ideally suited to"'(1996, p. 1).

Since the beginning of automation, the role of the human operator has changed, as automation takes over simple and repetitive work tasks, e.g., that it can do faster and more effectively than humans. The human operator's role has become to monitor the automation and to step in if it fails or to handle complex situations that the automation cannot handle. If the symbiosis between the human operator and automation is faulty, the operator can risk catching the out-of- 
the-loop syndrome or be overloaded with information. Thus, not be aware of what the automation is doing - leading to human error (Endsley, 1996).

There are serval definitions of human error. Don Norman (2013) divides human errors into mistakes, slips, and lapses. Mistakes are when the goal or the plan to reach the goal is wrong. For slips the intention is correct, but the action is wrong. Lapses occur when the user does not act at all and automation usually prevent it (Norman, 2013; Porathe et al., 2018). Endsley and Jones (2012) and Norman (2013) remark that the term human error should no longer be utilized. Instead, one should use design-induced errors, as the systems are often poorly designed for the operators physical and mental limits. Usually, the operator is not at fault when human errors occur; he is doing his best within the system that is designed. Human error is not only because operators do something wrong, but also due to the pressure in the competition or having too few crew members that causes fatigue (Allianz Global Corporate \& Specialty SE, 2013). With the commercial pressure in shipping today, tight financial conditions and time pressure are contributing factors of human errors. The pressure of being at the port in time and holding costs down creates an environment where taking risks is rewarded, creating a normalization of risk (AGSG, 2018; Rothblum, 2000).

\subsection{Human error and unmanned autonomous vessels}

The shipping industry has become safer because ships have more highly technical instruments and automation to help them maneuver than before (AGCS, 2018; Porathe et al., 2018). Still, the rate of accidents attributed to being caused by human error is still significant. According to the annual report by Allianz Global Cooperate \& Specialty (AGCS) on safety and shipping (2018) and as mentioned in the introduction, human error is estimated to be the cause of $75 \%$ to $96 \%$ of marine accidents. The unmanned autonomous vessel is predicted to reduce existing human errors considerably by reducing human shortcomings like fatigue, attention span, information overload and normality bias (Porathe et al., 2018). Thus, being safer than today's ships.

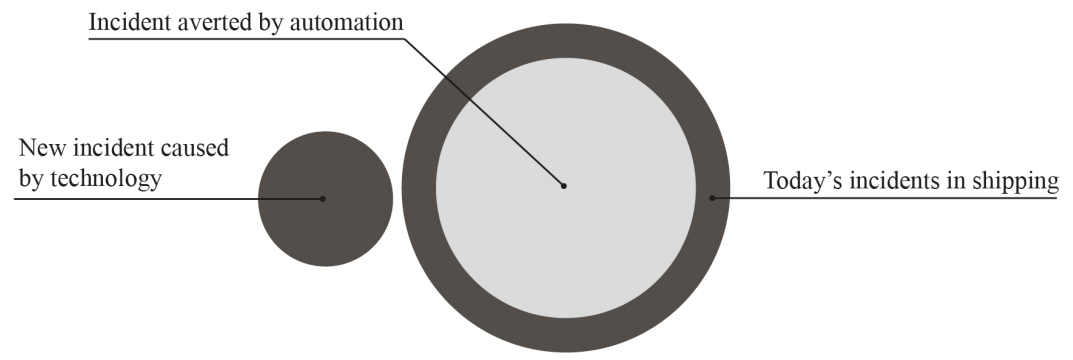

Figure 1. Human error incidents after the introduction of unmanned autonomous vessels, customized based on an original from Porathe et al., (2018).

Still there is concern that there will be new kinds of human error. From the design of the algorithms to the operator monitoring in the SCC. This will lead to even more significant and more catastrophic accidents. Porathe et al. (2018) voiced their concern that new technology and a complex system will create new kinds of human errors, even though the automation will reduce the human errors we experience today considerably (Figure 1). They concluded that human error still will be one of the main challenges in the development of unmanned autonomous vessels since it is human to err. 


\section{Human-Centred Design}

Human-centered design (HCD) is a method where the user is in the center for the development of new products, services, or systems instead of, e.g., technology-driven design, where the user must adapt to the system. By making use of the operators' pre-existing behavior in an HCD approach, one can create a more usable and robust system (Oviatt, 2006). The HCD approach focuses on an iterative process where the end user is a part of the whole process, from the inspiration phase to the ideation phase to the implementation phase (Ideo.org, 2018). One should be asking who, what, when, in what way and why when developing a new service, product or system (Giacomin, 2014). HCD has been around for a long time, having roots in ergonomics, computer science and artificial science (Giacomin, 2014). The International Organization for Standardization (ISO) have set some standard for HCD. Where ISO 9241-210, describes HCD as an "approach to systems design and development that aims to make interactive systems more usable by focusing on the use of the system and applying human factors, ergonomics and usability knowledge and techniques" (International Organization for Standardization 2010:2 as referred to in Giacomin, 2014, p. 608)

The International Marine Organization (IMO) sets standards for international shipping regarding safety, security and environmental performance (International maritime organization [IMO], n.d.). In 2015 HCD was added to the IMO guidelines for the development of enavigation systems. They recommend that the shipping industry should ensure that user needs are met through an HCD approach, as well as ensuring the trustworthiness of the software (IMO, 2015). An HCD approach is viewed as the right way for the shipping industry to go, as it may increase efficiency and effectiveness, decrease errors and accidents. Furthermore, an HCD approach will decrease training cost, personnel injuries and lost time (Rothblum, 2000). The IMO guidelines (2015) state that systems should be designed to improve user performance through all the systems life cycles and support the user in low and high workload environments. Design the system to fit the users' needs, instead of requiring users to adapt to the system (IMO, 2015). Usability testing is a useful tool to ensure that the system is developed and designed to fit the users need. The methods the IMO guidelines (2015) recommends conducting in usability tests, based on the ISO/TR 16982, are observation of users, performance related measurement, critical incident analysis, questionnaires, interviews and think aloud.

\section{Design Guidelines}

This paper has looked at how automation can challenge the operators' SA in different domains, and how a loss of SA can cause accidents attributed to human errors. One of the goals of developing unmanned autonomous vessels is to reduce these human errors, one way to reach this goal is to use HCD methods and taking the operators' mental capacity and SA into the design. By using affinity mapping to group the possible challenges the operators can face in a SCC, it was possible to see a pattern in the SA challenges. The main challenges for the operators SA are the automation, their mental capacity and relocating the operator form sea to shore. Based on the findings from the literature review, and the 50 design principles on how to design for better SA that Endsley and Jones (2012) presents in their book Designing for Situation Awareness, five design guidelines where made to approach the these challenges. The five design guidelines on how to design for the operators' SA in the SCC is presented in this section. 


\subsection{In the loop}

The SCC should be designed to keep the operator in the loop.

Being out-of-the-loop can result in making even the smallest automation failure catastrophic (Endsley \& Jones, 2012, p. 188). Automation today can take over monitoring and decision tasks for the operator, leaving him to have a passive decision making. Passive decision making is one of the causes of out-of-the-loop performance (Endsley \& Kiris, 1995). By making the operator take an active part in decisions making and increase the operator's involvement with the automation, designers can minimize the out-of-the-loop effect (Endsley \& Jones, 2012).

\subsection{Sensory information}

The SCC should be designed to replace sensory information that is no longer available for the operator.

Moving the control of the vessel from sea to shore, the operator no longer has the sensory information from the vessel's movements in the sea or feeling the weather on his skin. Parts of this sensory information is crucial in the creation of the operator's SA and must be replaced in the SCC in such a way that it does not degrade the operator's SA. There is a need for more research on this area, but by engaging users who have experience from manned ships in the design process of the SCC, one can ensure that the information available is enough to gain a good SA.

\subsection{Available information}

The SCC should provide enough information for the operator to obtain SA - without being overwhelmed.

The unmanned autonomous vessel's sensors will create an extensive amount of data. The rapity of this data surpasses the operator's ability to comprehend it, creating a cognitive bottleneck. The designer needs to identify what information is relevant for the operator and tailor the system design to fit those needs (Endsley \& Jones, 2012). To obtain a good SA with the staggering amount of data Endsley and Jones (2012) recommends organizing information around goals and double check with the operator to make sure that he obtains SA. The SCC should provide the operator with information that provide support for SA rather than decisions, this will enable the operator to see more than one solution to the task rather than accepting the solution the automation provided (Endsley \& Jones, 2012).

\subsection{Transparency}

\section{The SCC should provide automation transparency.}

The operator needs to know what the system is doing. By making the automation's current and future action transparent the operator will avoid surprises and misunderstandings. Transparency will help reduce the amount of automation-induced accidents where operators have misunderstood what mode the automation is set on (Endsley \& Jones, 2012). To achieve this transparency, designers can showcase the automation's goal or for example, showcase the expected route on a navigational map. 


\subsection{Automation level}

The SCC should indicate which level of autonomy the unmanned autonomous vessels operate on at all times.

The vessel's level of autonomy will vary during the voyage (Porathe et al., 2018). Misunderstanding what level of autonomy, the vessel is operating on can lead to accidents, where the operator believed the automation was in charge but in reality, the operator was the one in control. To avoid misunderstanding of what level of autonomy the vessel is operating on, designers should make sure that the level is always displayed to the operator

\section{Discussion}

Unmanned autonomous vessel has only just started being researched on, and it seems to be a push in the industry to be the first in developing a working unmanned autonomous vessel. The MUNIN project revealed that the technology is already available and made recommendations on how to develop an SCC (Porathe et al., 2014). Literature suggest that the introduction of unmanned autonomous vessels will improve maritime safety by reducing accidents caused by human error considerably. Still, literature also express concern that new, more complex errors will appear as the systems get more complex. Human errors will be present in the decisionmaking algorithms and in the SCCs, where operators will monitor and control the vessels. By taking use of HCD methods in the development of unmanned autonomous vessels and SCCs and designing for SA, one can minimize the development of new human errors.

No unmanned autonomous vessel or SCC exist today, but there are some under development that will finish in a couple of year. Because of this, there are no studies yet on how to operators handle the role of monitoring, or how often they must take control over the vessel. This paper has focused on how one can reduce human error in this new technology, by looking at other areas that have been studied more. Such as how automation affects SA as well as remote control of unmanned vehicles. The literature is consistent on that one has to consider the human limits and develop for the user. Based on a comprehensive literature review, this paper suggests five design guidelines to guide the design of a SCC that is optimal for the operators' SA. The guidelines are based on what the literature points out as the main causes for reduced SA, when looking at the combination of operators' SA and automation. To validate, the guidelines should be part of an iterative process since the knowledge of SCC and unmanned autonomous vessels still are under development. As the knowledge increases and possibility new challenges appears for the operator, the guidelines should change or be added to, to fit the new challenges and knowledge. As the field of unmanned autonomous vessels and SCC grows, there will possibly be a need for even more specific guidelines for the designers and developers.

Moving the operator from sea to shore will result in big changes for the operator who will be going from being a shipmaster to be an operator that supervises and monitors vessels in the SCC. The operator will no longer be out on the sea, feeling the motion of the ship beneath his feet, the weather on his skin and be able to look out and "read the sea". Designers must solve this loss of sensory information and design a solution that supports the operator's mental limits. On shore, the operator will likely be presented with visual and auditory information when monitoring one or more unmanned autonomous ships. Visual information can overload the operator's processing capacity, however, by designing the available information around goals the visual overload can be reduced. 
The unmanned autonomous vessel will be a complex system with large amounts of information available. Enhancing the operator's SA will be a challenge in the design of the system. Including the operators in this process and learn from their experience on manned ship will be crucial. The automation needs to be transparent in what it is doing and there should be enough information available to the operator to gain an overview of the situation. Systems that are designed without much thought on what information the operator need to access, will either hide critical information for the operator or it will be display it in a way that it is not easy to interpret. This may lead to misinterpretation of the system and consequently to poor decision making. As Endsley and Jones (2012, p. 164) stated: Automation is at its best when it has been "developed with high degree of ratability and fairly simple requirements for the human operator", but if it gets to complex the symbiosis of human and automation does not cooperate well. The need to involve users in the design process should be high on the priority list for developers, and should be conveyed to them by the designers involved.

\section{Conclusion}

Replacing technology-driven design with human-centered design, the human error will not only decrease but it also gives the opportunity to iterate the design of the SCC. With the ability to iterate one can change the design of the system, should one discover that operators struggle to obtain SA.

The unmanned autonomous vessels or its SCCs does not yet exist but are under development. By making use of the HCD approach in the developments period and involving the operators, one can avoid costly mistakes or misunderstandings. Still, time will show how much the unmanned autonomous vessels and the design of the SCC will reduce human error within shipping.

\section{Citations and References}

Allianz Global Corporate \& Specialty SE. (2013). Safety and Shippin 1912 - 2012: from Titanic to Costa Concordia. Retrieved from www.agcs.allianz.com

Allianz Global Corporate \& Specialty SE. (2018). Safety and Shipping Review 2018. Germany. Retrieved from www.agcs.allianz.com

Endsley, M. R. (1988). Design and evaluation for situation awareness enhancment. Proceedings of the Human Factors and Ergonomics Society Annual Meetin, Vol 32(2), 97-101. https://doi.org/https://doi.org/10.1177/154193128803200221

Endsley, M. R. (1995). A Taxonomy of Situation Awareness Errors. In Human Factors in Aviation Operations (pp. 287-292). Aldershot, England: Abebury Aviation: A.

Endsley, M. R. (1996). Automation and Situation Awareness. Automation and Human Performance: Theory and Applications, 163-181. https://doi.org/10.1177/154193120104500235

Endsley, M. R., \& Jones, D. G. (2012). Designing for Situation Awarness: An Approach to User-Centered Design (Second). CRC Press.

Endsley, M. R., \& Kiris, E. O. (1995). The Out-of-the-Loop Performance Problem and Level of Control in Automation. In Human Factors (Vol. 37, pp. 381-394). https://doi.org/10.1518/001872095779064555

Giacomin, J. (2014). What is human centred design? Design Journal, 17(4), 606-623. https://doi.org/10.2752/175630614X14056185480186

Ideo.org. (2018). What is human-centered design? Retrieved March 23, 2019, from http://www.designkit.org/human-centered-design 
Inagaki, T. (2006). Design of human-machine interactions in light of domain-dependence of human-centered automation. Cognition, Technology and Work, 8(3), 161-167. https://doi.org/10.1007/s10111-006-0034-z

International maritime organization [IMO]. (n.d.). Introduction to IMO. Retrieved March 30, 2019, from http://www.imo.org

International maritime organization [IMO]. (2015). Guideline On Software Quality Assurance And Human-Centred Design For E-Navigation. Retrieved from http://www.ics.org.ir/Downloads/CLD/News/MSC.1-Circ.1512 - Guideline On Software Quality Assurance And Human-Centred Design For E-Navigation (Secretariat).pdf

Kongsberg Gruppen. (2017). Autonomous ship project, key facts about YARA Birkeland -

Kongsberg Maritime. Retrieved September 13, 2018, from

https://www.km.kongsberg.com/ks/web/nokbg0240.nsf/AllWeb/4B8113B707A50A4FC 125811D00407045?OpenDocument

Manning, S. D., Rash, C. E., LeDuc, P. A., Noback, R. K., \& McKeon, J. (2004). The role of Human Casual Factors in U.S Army Unmanned Aerial Vehicle Accidents. Fort Rucker Al: U.S Army Aeromedical Reseach Laboratory.

Norman, D. (2013). Human error? No bad design! In The Design of Everyday Things (2nd ed., pp. 162-216). Basic Books.

Oviatt, S. (2006). Human-Centered Design Meets Cognitive Load Theory: Designing Interfaces that Help People Think. In Proceedings of the 14th ACM international conference on Multimedia (pp. 871-880). Santa Barbara, CA, USA: ACM. https://doi.org/10.1145/1180639.1180831

Porathe, T., Hoem, Å., Johnsen, S., Rødseth, Ø., \& Fjørtoft, K. (2018). At least as safe as manned shipping? Autonomous shipping, safety and " human error ." Esrel 2018, (June).

Porathe, T., \& Man, Y. (2013). MUNIN -D7.3. Technical layout of SOC.

Porathe, T., \& Man, Y. (2014). MUNIN - D7.5: HMI layout for SOC.

Porathe, T., Prison, J., \& Man, Y. (2014). Situation awareness in remote control centres for unmanned ships. Human Factors in Ship Design \& Operation, (February), 1-9.

Rødseth, Ø. (2018). Autonome skip internasjonalt: hvordan ligger norge an? Oslo.

Rødseth, Ø. J., \& Nordahl, H. (2018). Definition of autonomy levels for merchant ships, Report from NFAS, Norwegian Forum for Autonomous Ships, 2017-08-04. https://doi.org/10.13140/RG.2.2.21069.08163

Rothblum, A. M. (2000). Human Error and Marine Safety. National Safety Council Congress and Expo, Orlando, FL, 7. Retrieved from http://www.bowles-langley.com

Stensvold, T. (2018). Yara Birkeland skal bygges i Norge. Retrieved October 5, 2018, from https://www.tu.no/artikler/yara-birkeland-skal-bygges-i-norge/442400

Tinlund Ryste, A.-M. (2018). Yara Birkeland - a global game changer. Retrieved February 26, 2019, from https://www.youtube.com/watch?v=nCQJHZlxI6g

Whitlock, C. (2014). When drones fall from the sky | The Washington Post. Retrieved October 1, 2018, from https://www.washingtonpost.com/sf/investigative/2014/06/20/whendrones-fall-from-the-sky/?utm_term=.6e1ae8840fe7

Wiegmann, D. A., \& Shappell, S. A. (2007). A Human Error Approach to Aviation Accident Analysis : The Human Factors Analysis and Classification System. Routledge. 